\title{
One step cyclocondensation of (thio) barbituric acid with chalcones in glacial acetic acid and phosphorous pentoxide, Part-II.
}

\author{
K. Akhter ${ }^{1 *}$, M. E. Halim ${ }^{1}$, S. M. Ahmed ${ }^{2}$, P. K. Sarkar ${ }^{1}$, F. Akter ${ }^{1}$, K. Jahan ${ }^{1}$, U. K. R. Romman ${ }^{1}$ and \\ M. G. Ahmed ${ }^{1}$ \\ ${ }^{I}$ Department of Chemistry, University of Dhaka, Dhaka-1000, Bangladesh \\ ${ }^{2}$ Department of Chemistry, American International University-Bangladesh (AIUB), 83/B, Road-4, Kemal Ataturk Avenue, \\ Banani, Dhaka-1213, Bangladesh
}

\begin{abstract}
A number of new 5, 7-diaryl-1,5-dihydro (or 1, 2, 3, 5-tetrahydro)- pyrano [2, 3-d] pyrimidin-2, 4-diones (or 2-thioxo-4-ones) (3a-g) have been synthesized in one-step by cyclocondensation of barbituric acid or thiobarbituric acid (1) with arylideneacetophenones (2a-d), in glacial acetic acid in the presence of phosphorous pentoxide. The structures of the compounds 3a-g have been determined by UV, IR, 1H NMR, 13C NMR, mass spectral data and elemental analyses. The compounds 3a-g do not seem to be available in the literature.
\end{abstract}

Keywords: Arylideneacetophenone; Barbituric acid; Thiobarbituric acid; Cyclocondensation

\section{Introduction}

Pyran derivatives are ordinary structural subunits in a variety of important natural products, including carbohydrates, alkaloids, polyether antibiotics, pheromones, and iridoids (Tietze et al., 1997). Uracil and its fused derivatives, such as pyrano [2,3-d] pyrimidines, pyrido [2,3-d] pyrimidines or pyrimido [4,5-d] pyrimidines are well recognized by synthesis as well as biological chemists. These annelated uracils have received considerable attention over the past years due to their wide range of biological activity (Senda et al., 1968; Levitt, 1982; O'Callaghan et al., 1983; Wrigglesworth et al., 1984). Compounds with these ring systems have diverse pharmacological properties such as antiallergic (Kitamura et al., 1984), antihypertensive (Furuya et al., 1994), cardiotonic (Heber et al., 1993), bronchiodilator (Coates, 1990), antitumour activity (Broom et al., 1976). Pyrano [2,3-d] pyrimidine is unsaturated six membered heterocycle which is formed by fusion of pyran and pyrimidine rings together, consisting of one oxygen atom at position number 8 and two nitrogen atoms at position number 1 and 3 respectively. If pyrano [2,3-d] pyrimidine moieties are clubbed into one molecule, then resultant compound enhances its pharmaceutical activity as abundant in biologically active compounds. The synthesis of pyrano [2,3-d] pyrimidines containing a pyran and an uracil ring poses significant synthetic challenges. Therefore, for the preparation of these complex molecules large efforts have been directed towards the synthetic manipulation of uracils. As a result, a number of methods for the synthesis of these compounds have been reported (Rao et al., 1974; Junek et al., 1973; Noboru et al., 1973; Bararjanian et al., 2009; Ziarani et al., 2013), but the majority of them involve various steps, waste of organic solvents, long reaction time and the yields are relatively poor. This initiated to develop an efficient method for the synthesis of these compounds in better yields. Thus new routes for the synthesis of these molecules have attracted considerable attention in search for a rapid entry to these heterocycles. There is a report (Ahlwalia et al., 1993) on the reactions of barbituric acids with $a, \beta$-unsaturated carbonyl systems.

With this background, in continuation to our works (Ahmed et al., 2006; Ahmed et al., 2011; Ullah et al., 2012; Rahman et al., 2013; Akhter et al., 2016) on the synthesis of barbituric acid and thio-barbituric acid derivatives, we report herein syntheses of 5, 7-diaryl-1,5-dihydro - pyrano [2, 3-d] pyrimidin-2, 4-diones (3b, 3d \& 3e) and 5, 7-diaryl-2-thioxo - 1, 2, 3, 5-tetrahydro- pyrano [2, 3- $d$ ] pyrimidin -4-ones) (3a, 3c, $3 \mathrm{f} \& 3 \mathrm{~g}$ ) by selecting a number of arylideneacetophenones (2a-d) as the $a, \beta$-unsaturated carbonyl system having different substituents on the aromatic 
rings for reaction with barbituric acid or thiobarbituric acid (1) as the active methylene component in presence of glacial acetic acid and phosphorus pentoxide (Scheme 1). The compounds 3a-g do not seem to be available in the literature.

\section{Materials and methods}

The UV spectra were run in methanol using SHIMADZU-UV-160A ultraviolet spectrophotometer with a scanning range of $800-200 \mathrm{~nm}$ using methanol as solvent. The IR spectra were recorded as $\mathrm{KBr}$ pellet using SHIMADZU FT-IR $8400 \mathrm{~S}$ infra-red spectrophotometer in the range of $4000-400 \mathrm{~cm}^{-1}$. The ${ }^{1} \mathrm{H}$ - and ${ }^{13} \mathrm{C}$ - NMR spectra were recorded on $600 \mathrm{MHz}$ NMR spectrometer. The solvent used was $\mathrm{d}_{6}-$ DMSO and TMS was used as a reference. All the compounds gave expected $\mathrm{C}, \mathrm{H}$ and $\mathrm{N}$ analyses. 3-(2-methoxyphenyl)-1-(4-methylphenyl)-propenone $\mathbf{2 a}$, 3-(2-methoxyphenyl)-1-(4-chlorophenyl)-propenone 2b, 3-(4-methylphenyl)-1-phenyl-propenone 2c and 3-(4-methylphenyl)-1-(4-nitrophenyl)-propenone 2d were prepared from the reactions of corresponding substituted aldehydes and substituted acetophenones by following a literature method (Furniss et al., 1978) with modification wherever necessary. The reactions described in the present paper were carried out following a general procedure (Ahlwalia et al., 1993).

General procedure: A mixture of arylideneacetophenone $(0.005 \mathrm{~mol})$ and barbituric acid or thiobarbituric acid $(0.005$ mol) were dissolved in acetic acid $(15 \mathrm{~mL})$ and $\mathrm{P}_{2} \mathrm{O}_{5}(1.5 \mathrm{~g})$ in a round-bottomed flask equipped with a magnetic stirrer, a reflux condenser and a drying tube. The reaction mixture was refluxed at $130-140^{\circ} \mathrm{C}$ for $6-10$ hours and the course of the reaction was followed by TLC on silica gel plates. The mixture was allowed to cool and treated with crushed ice. The solid, thus obtained, was filtered off, washed with cold water, dried and purified by recrystallization from rectified spirit.

5-(2-Methoxyphenyl)-2-thioxo-7-p-tolyl-1,2,3,5-tetrahydr o-pyrano[2,3-d]pyrimidin-4-one, 3a: Light brown solid; Yield $46 \%$; mp. $230-232^{\circ} \mathrm{C} ; \mathrm{R}_{\mathrm{f}} 0.68\left(\mathrm{CHCl}_{3}\right.$ :Pet-ether $\left.=1: 4\right)$; $\mathrm{UV}: \lambda_{\text {max }} \mathrm{nm} 301\left(\pi \rightarrow \pi^{*} / \mathrm{n} \rightarrow \pi^{*}\right.$ of $\left.\mathrm{C}=\mathrm{O}\right)$; IR: $\gamma_{\text {max }}\left(\mathrm{cm}^{-1}\right) 3451$ $(\mathrm{N}-\mathrm{H}), 1677(\mathrm{C}=\mathrm{O}$, non-conj.), 1636, $1562(\mathrm{C}=\mathrm{O}$ arom, $\mathrm{C}-\mathrm{N})$ 1463, 1364, 1274, 1218, 1189, 1049, 1028, 949, 912, 860,
$814\left(\mathrm{C}=\mathrm{C}\right.$, arom. \& bar. acid moieties), 1137 (C-O-C); ${ }^{1} \mathrm{H}$

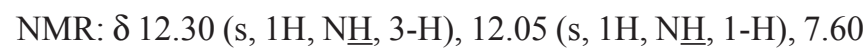
(d, $\left.\mathrm{J}_{8.0^{\prime}} 2 \mathrm{H}, \mathrm{H}-2^{\prime}, 6^{\prime}\right), 7.22$ (d, $\left.\mathrm{J}_{8.0^{\prime}}, 2 \mathrm{H}, \mathrm{H}-3^{\prime}, 5^{\prime}\right), 6.84-7.18$ (m, $\left.4 \mathrm{H}, \mathrm{H}-2^{\prime \prime}, 3^{\prime \prime}, 5^{\prime \prime}, 6^{\prime \prime}\right), 5.93\left(\mathrm{~d}, \mathrm{~J}_{4.5^{\prime}} 1 \mathrm{H}, 6-\mathrm{H}\right), 4.40\left(\mathrm{~d}, \mathrm{~J}_{4.5^{\prime}} 1 \mathrm{H}\right.$,

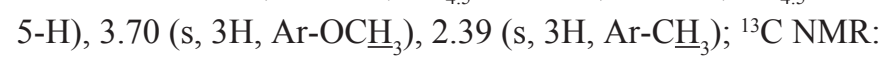

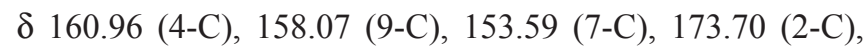
$144.94,138.79,136.03,129.15,128.85,128.49,124.10$, 113.80 (aromatic carbons), 103.26 (6-C), 92.92 (10-C), 33.91 (5-C), $55.07\left(\mathrm{Ar}-\mathrm{O} \underline{C H}_{3}\right), 20.79\left(\mathrm{Ar}-\underline{\mathrm{CH}}_{3}\right) ; \mathrm{MS}: \mathrm{m} / \mathrm{z} 378.14$ $\left(\mathrm{M}^{+}\right)$, 307.3, 289.07, 274.89, 154.1 (100\%), 136.2, 89.3; Anal. Found: C, 66.55; H, 4.64; N, 7.44; Calcd. for $\mathrm{C}_{21} \mathrm{H}_{18} \mathrm{~N}_{2} \mathrm{O}_{3} \mathrm{~S}$ : C, 66.65; H, 4.79; N, 7.40\%.

5-(2-Methoxyphenyl)-7-p-tolyl-1,5-dihydro-pyrano[2,3-d] pyrimidine-2,4-dione, 3b: Brown solid; Yield 43\%; mp. 204-206 ${ }^{\circ} \mathrm{C} ; \mathrm{R}_{\mathrm{f}} 0.68\left(\mathrm{CHCl}_{3}:\right.$ Pet-ether =1:4); UV: $\lambda_{\max } \mathrm{nm} 289$ $\left(\pi \rightarrow \pi^{*} / \mathrm{n} \rightarrow \pi^{*}\right.$ of $\left.\mathrm{C}=\mathrm{O}\right)$; IR: $\gamma_{\max }\left(\mathrm{cm}^{-1}\right) 3443(\mathrm{~N}-\mathrm{H}), 1690$ ( $\mathrm{C}=\mathrm{O}$, non-conj.), 1640, 1599, $1497(\mathrm{C}=\mathrm{O}$ arom, $\mathrm{C}-\mathrm{N})$ 1462, 1353, 1186, 1028, 968, 819, $755(\mathrm{C}=\mathrm{C}$, arom. \& bar. acid moieties), 1116 (C-O-C); ${ }^{1} \mathrm{H}$ NMR: $\delta 10.80$ (s, $\left.1 \mathrm{H}, \mathrm{N} \underline{\mathrm{H}}, 3-\mathrm{H}\right)$, 10.35 (s, 1H, N $\underline{\mathrm{H}}, 1-\mathrm{H}), 7.55$ (d, $\left.\mathrm{J}_{8.0}, 2 \mathrm{H}, \mathrm{H}-2^{\prime}, 6^{\prime}\right), 7.20$ (d, $\mathrm{J}_{8.0^{\prime}}$ 2H, H-3, 5), 6.80-7.19 (m, 4H, H-2", 3", 5", 6"), 5.99 (d, $\left.\mathrm{J}_{4.2^{\prime}} 1 \mathrm{H}, 6-\mathrm{H}\right), 4.45$ (d, $\left.\mathrm{J}_{4.2^{\prime}} 1 \mathrm{H}, 5-\mathrm{H}\right), 3.71$ (s, 3H, Ar-OC $\left.\underline{H}_{3}\right)$, $2.40\left(\mathrm{~s}, 3 \mathrm{H}, \mathrm{Ar}-\mathrm{C}_{3}\right)$; ${ }^{13} \mathrm{C}$ NMR: $\delta 163.36$ (4-C), 154.35 (9-C), 144.28 (7-C), 143.42 (2-C), 144.04, 138.90, 136.12, $129.35,128.60,128.21,123.81,113.15$ (aromatic carbons), 104.26 (6-C), 92.82 (10-C), 34.01 (5-C), $55.71\left(\mathrm{Ar}-\mathrm{OCH}_{3}\right)$, $20.80\left(\mathrm{Ar}^{-} \mathrm{CH}_{3}\right) ; \mathrm{MS}: \mathrm{m} / \mathrm{z} 362.20\left(\mathrm{M}^{+}\right), 307.11,289.3,154.0$ (100\%), 136.2, 89.1; Anal. Found: C, 69.50; H, 5.00; N, 7.63; Calcd. for $\mathrm{C}_{21} \mathrm{H}_{18} \mathrm{~N}_{2} \mathrm{O}_{4}$ : C, 69.60; H, 5.01; N, 7.73\%.

7-(4-Chlorophenyl)-5-(2-methoxyphenyl)-2-thioxo-1,2,3,5 -tetrahydro-pyrano[2,3- $d$ ]pyrimidine-4-one, 3c: Light purple solid; Yield 41\%; mp. $244-246^{\circ} \mathrm{C} ; \quad \mathrm{R}_{\mathrm{f}} \quad 0.73$ $\left(\mathrm{CHCl}_{3}:\right.$ Pet-ether $\left.=2: 3\right)$; UV: $\lambda_{\text {max }} \mathrm{nm} 431,300\left(\pi \rightarrow \pi^{*} / \mathrm{n} \rightarrow *\right.$ of $\mathrm{C}=\mathrm{O})$; IR: $\gamma_{\max }\left(\mathrm{cm}^{-1}\right) 3446(\mathrm{~N}-\mathrm{H}), 1680(\mathrm{C}=\mathrm{O}$, non-conj.), $1631,1589,1510(\mathrm{C}=\mathrm{O}$ arom, C-N $) 1461,1251,1178,1012$, $829(\mathrm{C}=\mathrm{C}$, arom. \& bar. acid moieties), 1131, 1092 (C-O-C);

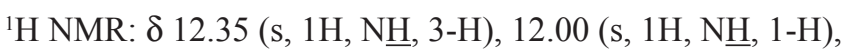
$7.70\left(\mathrm{~d}, \mathrm{~J}_{8.0^{\prime}} 2 \mathrm{H}, \mathrm{H}-2^{\prime}, 6^{\prime}\right), 7.55$ (d, $\left.\mathrm{J}_{8.0}, 2 \mathrm{H}, \mathrm{H}-3^{\prime}, 5^{\prime}\right)$, 6.98-7.20 (m, 4H, H-2",3", 5",6"), 5.88 (d, J.4. $1 \mathrm{H}, 6-\mathrm{H}), 4.49$ $\left(\mathrm{d}, \mathrm{J}_{4.4^{\prime}} 1 \mathrm{H}, 5-\mathrm{H}\right), 3.69$ (s, 3H, Ar-OCH$\left.{ }_{3}\right) ;{ }^{13} \mathrm{C}$ NMR: $\delta 161.96$ (4-C), 155.43 (9-C), 145.01 (7-C), 173.70 (2-C), 145.92, 
$139.44,132.90,130.12,129.77,128.55,128.22,123.31$, 114.10 (aromatic carbons), 103.72 (6-C), 88.45 (10-C), 32.85 (5-C), $56.10\left(\mathrm{Ar}^{-} \mathrm{OCH}_{3}\right)$; MS: m/z $398.06\left(\mathrm{M}^{+}\right), 307.6$, 289.1, 154.1 (100\%), 136.0, 89.2; Anal. Found: C, 60.30; H, 3.69; N, 7.09; Calcd. for $\mathrm{C}_{20} \mathrm{H}_{15} \mathrm{~N}_{2} \mathrm{O}_{3} \mathrm{ClS}$ : C, 60.22; H, 3.79; $\mathrm{N}, 7.02 \%$.

7-(4-Chlorophenyl)-5-(2-methoxyphenyl)-1,5-dihydro-py rano[2,3-d]pyrimidine-2,4-dione, 3d: Brown solid; Yield $38 \%$; mp. 216-218 ${ }^{\circ}$; $\mathrm{R}_{\mathrm{f}} 0.67\left(\mathrm{CHCl}_{3}\right.$ :Pet-ether $\left.=2: 3\right)$; UV:

$\lambda_{\max } \mathrm{nm} 293\left(\pi \rightarrow \pi^{*} / \mathrm{n} \rightarrow \pi^{*}\right.$ of $\left.\mathrm{C}=\mathrm{O}\right) ;$ IR: $\gamma_{\max }\left(\mathrm{cm}^{-1}\right) 3463$ (N-H), 1691 (C=O, non-conj.), 1630, 1589, $1491(\mathrm{C}=\mathrm{O}$ arom, C-N) $1250,1181,1012,971,829,760(\mathrm{C}=\mathrm{C}$, arom. \& bar.

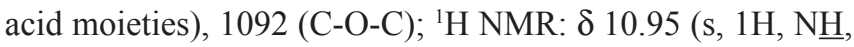

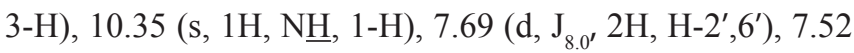
(d, J ${ }_{8.0^{\prime}}$ 2H, H-3', 5'), 6.95-7.22 (m, 4H, H-2",3",5",6"), 5.91 (d, $\left.\mathrm{J}_{4.1}, 1 \mathrm{H}, 6-\mathrm{H}\right), 4.45$ (d, $\left.\mathrm{J}_{4.1}, 1 \mathrm{H}, 5-\mathrm{H}\right), 3.70$ (s, 3H, Ar-OC $\left.\underline{H}_{3}\right)$; ${ }^{13}$ C NMR: $\delta 163.10$ (4-C), 155.41 (9-C), 145.91 (7-C), 143.70 (2-C), 144.44, 132.71, 130.11, 129.67, 128.53, 128.12, 123.21, 114.00 (aromatic carbons), 103.90 (6-C), 89.35 (10-C), 32.82 (5-C), $56.15\left(\mathrm{Ar}-\mathrm{OCH}_{3}\right) ; \mathrm{MS}: \mathrm{m} / \mathrm{z} 382.19\left(\mathrm{M}^{+}\right)$, 307.2, 289.0, 154.0 (100\%), 136.1, 89.2; Anal. Found: C, 62.70; H, 3.89; N, 7.22; Calcd. for $\mathrm{C}_{20} \mathrm{H}_{15} \mathrm{~N}_{2} \mathrm{O}_{4} \mathrm{Cl}$ : C, 62.75; H, 3.95; N, 7.32\%.

7-Phenyl-5-p-tolyl--1,5-dihydro-pyrano[2,3-d]pyrimidine -2,4-dione, 3e: Brown solid; Yield 51\%; mp. 246-248 ${ }^{\circ}$; $\mathrm{R}_{\mathrm{f}}$ $0.68\left(\right.$ Neat $\left.\mathrm{CHCl}_{3}\right)$; UV: $\lambda_{\max } \mathrm{nm} 315\left(\pi \rightarrow \pi^{*} / \mathrm{n} \rightarrow \pi^{*}\right.$ of $\left.\mathrm{C}=\mathrm{O}\right)$; IR: $\gamma_{\max }\left(\mathrm{cm}^{-1}\right) 3434(\mathrm{~N}-\mathrm{H}), 1699(\mathrm{C}=\mathrm{O}$, non-conj.), 1631, $1530(\mathrm{C}=\mathrm{O}$ arom, C-N $) 1446,1279,1251,1038,917,818$, $765\left(\mathrm{C}=\mathrm{C}\right.$, arom. \& bar. acid moieties), $1118(\mathrm{C}-\mathrm{O}-\mathrm{C}) ;{ }^{1} \mathrm{H}$

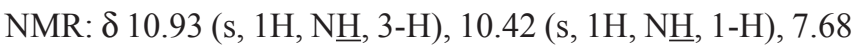
(d, $\left.\mathrm{J}_{8.2^{\prime}} 2 \mathrm{H}, \mathrm{H}-2^{\prime}, 6^{\prime}\right), 7.39$ (d, J $\left.{ }_{8.2^{\prime}} 2 \mathrm{H}, \mathrm{H}-3^{\prime}, 5^{\prime}\right), 7.09-7.14$ (m, $5 \mathrm{H}, \mathrm{H}-2^{\prime \prime}, 3^{\prime \prime}, 4^{\prime \prime}, 5^{\prime \prime}, 6$ ") 5.98 (bs,1H, 6-H), 4.41 (bs, 1H, 5-H), $2.24\left(\mathrm{~s}, 3 \mathrm{H}, \mathrm{Ar}-\mathrm{CH}_{3}\right) ;{ }^{13} \mathrm{C} \mathrm{NMR}: \delta 163.02$ (4-C), 155.08 (9-C), 149.63 (7-C), 144.59 (2-C), 139.79, 135.83, 131.88, $130.11,129.71,128.27,127.68,125.97$ (aromatic carbons), 102.67 (6-C), 85.94 (10-C), 32.44 (5-C), 21.13 (Ar- CH$\left._{3}\right)$; MS: m/z 332.25 (M+), 289.12, 238.11, 154.0 (100\%), 136.0, 89.3, 57.2; Anal. Found: C, 72.33; H, 4.70; N, 8.40; Calcd. for $\mathrm{C}_{20} \mathrm{H}_{16} \mathrm{~N}_{2} \mathrm{O}_{3}$ : C, 72.28; H, 4.85; N, 8.43\%.

7-Phenyl-2-thioxo-5-p-tolyl-1,2,3,5-tetrahydro-pyrano [2,3-d]pyrimidin-4-one, 3f: Brown solid; Yield 55\%; mp. $267-269^{\circ} \mathrm{C} ; \mathrm{R}_{\mathrm{f}} 0.74\left(\mathrm{Neat} \mathrm{CHCl}_{3}\right.$ ); UV: $\lambda_{\max } \mathrm{nm} 318$, $209\left(\pi \pi^{*} / \mathrm{n} \rightarrow \pi^{*}\right.$ of $\left.\mathrm{C}=\mathrm{O}\right)$; IR: $\gamma_{\max }\left(\mathrm{cm}^{-1}\right) 3400(\mathrm{~N}-\mathrm{H})$, 1700, 1650 ( $\mathrm{C}=\mathrm{O}$, non-conj.), 1590, $1555(\mathrm{C}=\mathrm{O}$ arom, C-N) 1430, 1320, 1215, 1180, 1030, 1005, $810(\mathrm{C}=\mathrm{C}$, arom. \& bar. acid moieties), 1131, 1078 (C-O-C); ${ }^{1} \mathrm{H}$

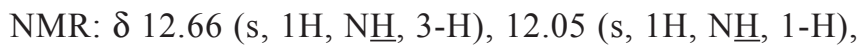
$7.70\left(\mathrm{~d}, \mathrm{~J}_{8.1}, 2 \mathrm{H}, \mathrm{H}-2^{\prime}, 6^{\prime}\right), 7.42\left(\mathrm{~d}, \mathrm{~J}_{8.1}, 2 \mathrm{H}, \mathrm{H}-3^{\prime}, 5^{\prime}\right)$, 7.02-7.19 (m, 5H, H-2",3",4",5",6"), 5.95 (bs, 1H, 6-H), $4.60(\mathrm{bs}, 1 \mathrm{H}, 5-\mathrm{H}), 2.26\left(\mathrm{~s}, 3 \mathrm{H}, \mathrm{Ar}-\mathrm{CH}_{3}\right) ;{ }^{13} \mathrm{C} \mathrm{NMR}: \delta$ 163.20 (4-C), 155.80 (9-C), 148.61 (7-C), 174.59 (2-C), $139.80,133.73,131.78,130.10,129.70,128.75,127.70$, 125.51 (aromatic carbons), 102.62 (6-C), 86.15 (10-C), 32.40 (5-C), $20.97\left(\mathrm{Ar}-\underline{\mathrm{CH}}_{3}\right) ; \mathrm{MS}: \mathrm{m} / \mathrm{z} 348.12\left(\mathrm{M}^{+}\right)$, 289.33, 154.0 (100\%), 136.2, 89.0; Anal. Found: C, 68.78; H, 4.90; N, 8.03; Calcd. for $\mathrm{C}_{20} \mathrm{H}_{16} \mathrm{~N}_{2} \mathrm{O}_{2} \mathrm{~S}$ : C, 68.94; $\mathrm{H}, 4.63 ; \mathrm{N}, 8.04 \%$.

7-(4-Nitrophenyl)-2-thioxo-5-p-tolyl-1,2,3,5-tetrahydro-p yrano[2,3-d]pyrimidin-4-one, 3g: Reddish brown solid; Yield 58\%; mp. $232-234^{\circ} \mathrm{C} ; \mathrm{R}_{\mathrm{f}} 0.66\left(\mathrm{CHCl}_{3}\right.$ :Pet-ether $\left.=4: 1\right)$; UV: $\lambda_{\max }$ nm 335, $213\left(\pi \rightarrow \pi^{*} / \mathrm{n} \rightarrow \pi^{*}\right.$ of $\left.C=O\right)$; IR: $\gamma_{\max }\left(\mathrm{cm}^{-1}\right)$ $3446(\mathrm{~N}-\mathrm{H}), 1665$ (C=O, non-conj.), 1598, $1518(\mathrm{C}=\mathrm{O}$ arom, C-N) 1345, 1206, 1032, 853, 812, $751(\mathrm{C}=\mathrm{C}$, arom. \& bar.

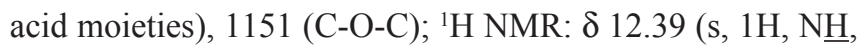

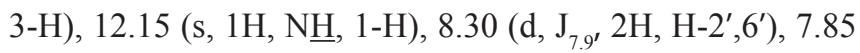
(d, J.9' $\left.2 \mathrm{H}, \mathrm{H}-3^{\prime}, 5^{\prime}\right), 6.98-7.29$ (m, 4H, H-2",3",5",6"), 5.99 (d, J $\left.{ }_{3.9^{\prime}} 1 \mathrm{H}, 6-\mathrm{H}\right), 4.50$ (d, J $\left.{ }_{3.9^{\prime}} 1 \mathrm{H}, 5-\mathrm{H}\right), 2.35$ (s, 3H, Ar- $\left.\underline{\mathrm{H}}_{3}\right)$; ${ }^{13}$ C NMR: $\delta 165.40$ (4-C), 154.50 (9-C), 148.60 (7-C), 175.59 (2-C), 149.53, 142.80, 134.50, 130.87, 129.70, 128.75, 127.28, 123.77 (aromatic carbons), 105.65 (6-C), 89.65 (10-C), 34.44 (5-C), $21.70\left(\mathrm{Ar}^{-} \underline{\mathrm{CH}}_{3}\right) ; \mathrm{MS}: \mathrm{m} / \mathrm{z} 393.22\left(\mathrm{M}^{+}\right)$, 383.0 (100\%), 271.0, 256.0, 135.0, 88.4; Anal. Found: C, 61.14; H, 3.98; N, 10.64; Calcd. for $\mathrm{C}_{20} \mathrm{H}_{15} \mathrm{~N}_{3} \mathrm{O}_{4} \mathrm{~S}: \mathrm{C}, 61.06$; $\mathrm{H}, 3.84 ; \mathrm{N}, 10.68 \%$.

\section{Results and discussion}

The Compounds 3a-g have been synthesized from $\mathbf{1}$ and the corresponding $\mathbf{2 a - d}$ in presence of glacial acetic acid and $\mathrm{P}_{2} \mathrm{O}_{5}$ under refluxing conditions in an analogous manner reported previously (Ahlwalia et al., 1993). The Compounds 3a-g have been characterized on the basis of their UV/Vis, IR, ${ }^{1} \mathrm{H}$ NMR, ${ }^{13} \mathrm{C}$ NMR, mass and elemental analyses. The formation of compounds $\mathbf{3 a - g}$ may be explained by the initial formation of a 1:1 adduct (A) followed by cyclocondensation (Scheme 1). The formation of such an adduct has been reported (Kharchenko et al., 1976) in the literature. 


\section{Scheme 1: Synthesis of Pyrano[2,3-d]pyrimidines}<smiles>[Z]C1NC(=O)CC(=O)N1</smiles>

1

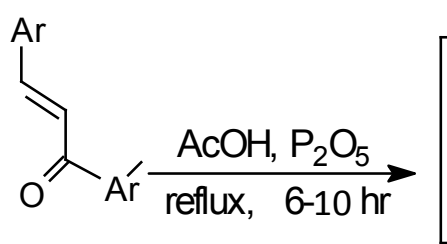

2

$\begin{array}{clll}\text { Products } & \mathrm{Ar} & \mathrm{A}^{\prime} \mathrm{r} & \mathrm{Z} \\ \text { 3a } & 2-\mathrm{CH}_{3} \mathrm{OC}_{6} \mathrm{H}_{5} & 4-\mathrm{CH}_{3} \mathrm{C}_{6} \mathrm{H}_{5} & \mathrm{~S} \\ 3 \mathrm{~b} & 2-\mathrm{CH}_{3} \mathrm{OC}_{6} \mathrm{H}_{5} & 4-\mathrm{CH}_{3} \mathrm{C}_{6} \mathrm{H}_{4} & \mathrm{O} \\ 3 \mathrm{c} & 2-\mathrm{CH}_{3} \mathrm{OC}_{6} \mathrm{H}_{5} & 4-\mathrm{ClC}_{6} \mathrm{H}_{4} & \mathrm{~S} \\ 3 \mathrm{~d} & 2-\mathrm{CH}_{3} \mathrm{OC}_{6} \mathrm{H}_{5} & 4-\mathrm{ClC}_{6} \mathrm{H}_{4} & \mathrm{O} \\ 3 \mathrm{e} & 4-\mathrm{CH}_{3} \mathrm{C}_{6} \mathrm{H}_{5} & 4-\mathrm{C}_{6} \mathrm{H}_{5} & \mathrm{O} \\ 3 \mathrm{f} & 4-\mathrm{CH}_{3} \mathrm{C}_{6} \mathrm{H}_{5} & 4-\mathrm{C}_{6} \mathrm{H}_{5} & \mathrm{~S} \\ 3 \mathrm{~g} & 4-\mathrm{CH}_{3} \mathrm{C}_{6} \mathrm{H}_{5} & 4-\mathrm{O}_{2} \mathrm{NC}_{6} \mathrm{H}_{4} & \mathrm{~S}\end{array}$<smiles></smiles>

A<smiles>CC(C)O</smiles><smiles>[Z]c1nc2c(c(=O)[nH]1)[C@@H](Br)C=C([In])O2</smiles>

$3 a-9$

$(1: 1$ molarratio $)$

Table I. Reaction conditions and analytical data of the compounds 3a-g

\begin{tabular}{|c|c|c|c|c|c|c|c|}
\hline Compound & $\begin{array}{l}\text { Reflux } \\
\text { time } \\
\text { (hr) }\end{array}$ & $\begin{array}{l}\text { Reaction } \\
\text { temp. }\left({ }^{\circ} \mathrm{C}\right)\end{array}$ & $\begin{array}{l}\% \mathrm{C} \\
\text { Found } \\
\text { (Calcd) }\end{array}$ & $\begin{array}{l}\% \mathrm{H} \\
\text { Found } \\
\text { (Calcd) }\end{array}$ & $\begin{array}{l}\% \mathrm{~N} \\
\text { Found } \\
\text { (Calcd) }\end{array}$ & Mol. formula & $\mathrm{MS}(\mathrm{m} / \mathrm{z})$ \\
\hline $3 \mathbf{a}$ & 7 & 130 & $\begin{array}{l}66.55 \\
(66.65)\end{array}$ & $\begin{array}{l}4.64 \\
(4.79)\end{array}$ & $\begin{array}{l}7.44 \\
(7.40)\end{array}$ & $\mathrm{C}_{21} \mathrm{H}_{18} \mathrm{~N}_{2} \mathrm{O}_{3} \mathrm{~S}$ & 378.14 \\
\hline $\mathbf{3 b}$ & 8 & 140 & $\begin{array}{l}69.50 \\
(69.60)\end{array}$ & $\begin{array}{l}5.00 \\
(5.01)\end{array}$ & $\begin{array}{l}7.63 \\
(7.73)\end{array}$ & $\mathrm{C}_{21} \mathrm{H}_{18} \mathrm{~N}_{2} \mathrm{O}_{4}$ & 362.20 \\
\hline $3 c$ & 7.5 & 139 & $\begin{array}{l}60.30 \\
(60.22)\end{array}$ & $\begin{array}{l}3.69 \\
(3.79)\end{array}$ & $\begin{array}{l}7.09 \\
(7.02)\end{array}$ & $\mathrm{C}_{20} \mathrm{H}_{15} \mathrm{~N}_{2} \mathrm{O}_{3} \mathrm{ClS}$ & 398.06 \\
\hline 3d & 6.5 & 136 & $\begin{array}{l}62.70 \\
(62.75)\end{array}$ & $\begin{array}{l}3.89 \\
(3.95)\end{array}$ & $\begin{array}{l}7.22 \\
(7.32)\end{array}$ & $\mathrm{C}_{20} \mathrm{H}_{15} \mathrm{~N}_{2} \mathrm{O}_{4} \mathrm{Cl}$ & 382.19 \\
\hline $3 e$ & 6.5 & 140 & $\begin{array}{l}72.33 \\
(72.28)\end{array}$ & $\begin{array}{l}4.70 \\
(4.85)\end{array}$ & $\begin{array}{l}8.40 \\
(8.43)\end{array}$ & $\mathrm{C}_{20} \mathrm{H}_{16} \mathrm{~N}_{2} \mathrm{O}_{3}$ & 332.25 \\
\hline $3 f$ & 10 & 130 & $\begin{array}{l}68.78 \\
(68.94)\end{array}$ & $\begin{array}{l}4.90 \\
(4.63)\end{array}$ & $\begin{array}{l}8.03 \\
(8.04)\end{array}$ & $\mathrm{C}_{20} \mathrm{H}_{16} \mathrm{~N}_{2} \mathrm{O}_{2} \mathrm{~S}$ & 348.12 \\
\hline $3 g$ & 6 & 135 & $\begin{array}{l}61.14 \\
(61.06)\end{array}$ & $\begin{array}{l}3.98 \\
(3.84)\end{array}$ & $\begin{array}{l}10.64 \\
(10.68)\end{array}$ & $\mathrm{C}_{20} \mathrm{H}_{15} \mathrm{~N}_{3} \mathrm{O}_{4} \mathrm{~S}$ & 393.22 \\
\hline
\end{tabular}

In their UV spectra of compounds 3a-g the observed $\lambda_{\max }$ values agree well to the expected values. The absorption bands in the range 431-209 nm may be assigned to the $\pi \rightarrow \pi^{*}$ of $\mathrm{C}=\mathrm{O}$ in these compounds. The weak $\mathrm{n} \rightarrow \pi^{*}$ absorption bands in the cases of these compounds due to $\mathrm{C}=\mathrm{O}$ were probably masked within the $\pi \rightarrow \pi^{*}$ absorption range. 
Table II. Physical Constants, IR and UV of compounds 3a-g

\begin{tabular}{|c|c|c|c|c|c|c|c|c|c|}
\hline \multirow[b]{2}{*}{ Compou nd } & \multirow[b]{2}{*}{$\begin{array}{l}\text { m.p. } \\
\left({ }^{\circ} \mathrm{C}\right)\end{array}$} & \multirow[b]{2}{*}{$\begin{array}{l}\text { Yield } \\
(\%)\end{array}$} & \multirow[b]{2}{*}{$\mathrm{R}_{\mathrm{f}}$ value } & \multicolumn{5}{|c|}{$\mathrm{IR}, v_{\max }$ in $\mathrm{cm}^{-1}$} & \multirow{2}{*}{$\begin{array}{c}\mathrm{UV}, \lambda_{\text {max }} \\
\quad(\mathrm{nm}) \\
\pi \rightarrow \pi^{*}, \\
\mathrm{n} \rightarrow \pi^{*}\end{array}$} \\
\hline & & & & $\mathrm{N}-\mathrm{H}$ & $\begin{array}{l}\mathrm{C}=\mathrm{O} \\
\text { non- } \\
\text { conj. }\end{array}$ & $\begin{array}{l}\mathrm{C}=\mathrm{O} \\
\text { arom } \\
, \mathrm{C}-\mathrm{N}\end{array}$ & $\begin{array}{l}\quad \mathrm{C}=\mathrm{C} \\
\text { (arom. \& bar. } \\
\text { acid moieties) }\end{array}$ & $\mathrm{C}-\mathrm{O}-\mathrm{C}$ & \\
\hline $3 \mathbf{a}$ & $230-232$ & 46 & $\begin{array}{c}0.68 \\
\left(\mathrm{CHCl}_{3}:\right. \\
\text { Pet-ether }= \\
1: 4)\end{array}$ & 3451 & 1677 & $\begin{array}{l}1636 \\
1562\end{array}$ & $\begin{array}{c}1463,1364,1274 \\
1218,1189,1049 \\
1028,949,912 \\
860,814\end{array}$ & 1137 & 301 \\
\hline $\mathbf{3 b}$ & 204-206 & 43 & $\begin{array}{c}0.68 \\
\left(\mathrm{CHCl}_{3}:\right. \\
\text { Pet-ether }= \\
1: 4)\end{array}$ & 3443 & 1690 & $\begin{array}{l}1640 \\
1599\end{array}$ & $\begin{array}{c}1462,1353,1186 \\
1028,968,819 \\
755\end{array}$ & 1116 & 289 \\
\hline $3 c$ & $244-246$ & 41 & $\begin{array}{c}0.73 \\
\left(\mathrm{CHCl}_{3}:\right. \\
\text { Pet-ether }= \\
2: 3)\end{array}$ & 3446 & 1680 & $\begin{array}{l}1631 \\
1589\end{array}$ & $\begin{array}{c}1461,1251,1178 \\
1012,829\end{array}$ & $\begin{array}{l}1131 \\
1092\end{array}$ & 431,300 \\
\hline 3d & $216-218$ & 38 & $\begin{array}{c}0.67 \\
\left(\mathrm{CHCl}_{3}:\right. \\
\text { Pet-ether }= \\
2: 3)\end{array}$ & 3463 & 1691 & $\begin{array}{l}1630 \\
1589\end{array}$ & $\begin{array}{c}1250,1181,1012 \\
971,829,760\end{array}$ & 1092 & 293 \\
\hline $3 e$ & $246-248$ & 51 & $\begin{array}{c}0.68(\text { Neat } \\
\left.\mathrm{CHCl}_{3}\right)\end{array}$ & 3434 & 1699 & $\begin{array}{l}1631 \\
1530\end{array}$ & $\begin{array}{c}1446,1279,1251 \\
1038,917,818 \\
765\end{array}$ & 1118 & 315 \\
\hline $3 f$ & $267-269$ & 55 & $\begin{array}{c}0.74(\text { Neat } \\
\left.\mathrm{CHCl}_{3}\right)\end{array}$ & 3400 & $\begin{array}{l}1700 \\
1650\end{array}$ & $\begin{array}{l}1590 \\
1555\end{array}$ & $\begin{array}{c}1430,1320,1215 \\
1180,1030,1005 \\
810\end{array}$ & $\begin{array}{l}1131 \\
1078\end{array}$ & 318,209 \\
\hline $3 g$ & $232-234$ & 58 & $\begin{array}{c}0.66 \\
\left(\mathrm{CHCl}_{3}:\right. \\
\text { Pet-ether }= \\
4: 1)\end{array}$ & 3446 & 1665 & $\begin{array}{l}1598 \\
1518\end{array}$ & $\begin{array}{c}1345,1206,1032 \\
853,812,751\end{array}$ & 1151 & 335,213 \\
\hline
\end{tabular}<smiles>[X]c1ccc(C2=CC(c3ccccc3)C3=C(NC(=[Z])NC3=O)O2)cc1</smiles><smiles></smiles> 
The IR data of the compounds $\mathbf{3 a - g}$ (Table 2) showed sharp as well as broad bands in the range $\left(v_{\max }\right) 3463-3400$ $\mathrm{cm}^{-1}$ indicating the presence of $\mathrm{N}-\mathrm{H}$ group. The absorption bands at $1700-1650 \mathrm{~cm}^{-1}$ indicate the presence of non-conjugated $\mathrm{C}=\mathrm{O}$ stretching including the barbituric acid moieties (Bojarski et al., 1985). The bands at

Table III. ${ }^{1}$ H NMR spectral data of the compounds $3 \mathrm{a}-\mathrm{g} .[(\mathrm{d})$ in ppm]

\begin{tabular}{|c|c|c|c|c|c|c|c|}
\hline Compound & $3-\mathrm{H}$ & $1-\mathrm{H}$ & Aromatic & $6-\mathrm{H}$ & $5-\mathrm{H}$ & $\mathrm{X}$ & $\mathrm{Y}$ \\
\hline $3 a$ & $\begin{array}{l}12.30 \\
(\mathrm{~s}, 1 \mathrm{H}, \mathrm{N} \underline{\mathrm{H}})\end{array}$ & $\begin{array}{l}12.05 \\
(\mathrm{~s}, 1 \mathrm{H}, \mathrm{N} \underline{\mathrm{H}})\end{array}$ & $\begin{array}{l}7.60\left(\mathrm{~d}, \mathrm{~J}_{8.0}, 2 \mathrm{H}, \mathrm{H}-2^{\prime}, 6^{\prime}\right) \\
7.22\left(\mathrm{~d}, \mathrm{~J}_{8.0}, 2 \mathrm{H}, \mathrm{H}-3^{\prime}, 5^{\prime}\right) \\
6.84-7.18(\mathrm{~m}, 4 \mathrm{H}, \mathrm{H}- \\
\left.2^{\prime \prime}, 3^{\prime}, 5^{\prime}, 6^{\prime}\right)\end{array}$ & $\begin{array}{l}5.93 \\
\left(\mathrm{~d}, \mathrm{~J}_{4.5}, 1 \mathrm{H}\right)\end{array}$ & $\begin{array}{l}4.40 \\
\left(\mathrm{~d}, \mathrm{~J}_{4.5}, 1 \mathrm{H}\right)\end{array}$ & $\begin{array}{l}3.70 \\
(\mathrm{Ar}- \\
\left.\mathrm{OC}_{3}{ }_{3}\right)\end{array}$ & $\begin{array}{l}2.39 \\
(\mathrm{Ar}- \\
\left.\mathrm{C}_{3}\right)\end{array}$ \\
\hline $\mathbf{3 b}$ & $\begin{array}{l}10.80 \\
(\mathrm{~s}, 1 \mathrm{H}, \mathrm{N} \underline{\mathrm{H}})\end{array}$ & $\begin{array}{l}10.35 \\
(\mathrm{~s}, 1 \mathrm{H}, \mathrm{N} \underline{\mathrm{H}})\end{array}$ & $\begin{array}{l}7.55\left(\mathrm{~d}, \mathrm{~J}_{8.0}, 2 \mathrm{H}, \mathrm{H}-2^{\prime}, 6^{\prime}\right) \\
7.20\left(\mathrm{~d}, \mathrm{~J}_{8.0}, 2 \mathrm{H}, \mathrm{H}-3^{\prime}, 5^{\prime}\right) \\
6.80-7.19(\mathrm{~m}, 4 \mathrm{H}, \mathrm{H}- \\
\left.2 ”, 3^{\prime}, 5^{\prime}, 6^{\prime}\right)\end{array}$ & $\begin{array}{l}5.99 \\
\left(\mathrm{~d}, \mathrm{~J}_{4.2}, 1 \mathrm{H}\right)\end{array}$ & $\begin{array}{l}4.45 \\
\left(\mathrm{~d}, \mathrm{~J}_{4.2}, 1 \mathrm{H}\right)\end{array}$ & $\begin{array}{l}3.71 \\
(\mathrm{Ar}- \\
\left.\mathrm{OC}_{3}\right)\end{array}$ & $\begin{array}{l}2.40 \\
(\mathrm{Ar}- \\
\left.\mathrm{C}_{3}\right)\end{array}$ \\
\hline $3 c$ & $\begin{array}{l}12.35 \\
(\mathrm{~s}, 1 \mathrm{H}, \mathrm{N} \underline{\mathrm{H}})\end{array}$ & $\begin{array}{l}12.00 \\
(\mathrm{~s}, 1 \mathrm{H}, \mathrm{N} \underline{\mathrm{H}})\end{array}$ & $\begin{array}{l}7.70\left(\mathrm{~d}, \mathrm{~J}_{8.0}, 2 \mathrm{H}, \mathrm{H}-3^{\prime \prime}, 5^{\prime \prime}\right) \\
7.55\left(\mathrm{~d}, \mathrm{~J}_{8.0}, 2 \mathrm{H}, \mathrm{H}-2^{\prime}, 6^{\prime}\right) \\
6.98-7.20\left(\mathrm{~m}, 4 \mathrm{H}, \mathrm{H}-2^{\prime \prime},\right. \\
\left.3^{\prime}, 5^{\prime}, 6^{\prime \prime}\right)\end{array}$ & $\begin{array}{l}5.88 \\
\left(\mathrm{~d}, \mathrm{~J}_{4.4}, 1 \mathrm{H}\right)\end{array}$ & $\begin{array}{l}4.49 \\
\left(\mathrm{~d}, \mathrm{~J}_{4.4}, 1 \mathrm{H}\right)\end{array}$ & $\begin{array}{l}3.69 \\
(\mathrm{Ar}- \\
\left.\mathrm{OCH}_{3}\right)\end{array}$ & $\ldots$ \\
\hline $3 d$ & $\begin{array}{l}10.95 \\
(\mathrm{~s}, 1 \mathrm{H}, \mathrm{N} \underline{\mathrm{H}})\end{array}$ & $\begin{array}{l}10.35 \\
(\mathrm{~s}, 1 \mathrm{H}, \mathrm{N} \underline{\mathrm{H}})\end{array}$ & $\begin{array}{l}7.69\left(\mathrm{~d}, \mathrm{~J}_{8.0}, 2 \mathrm{H}, \mathrm{H}-3 ”, 5^{\prime \prime}\right) \\
7.52\left(\mathrm{~d}, \mathrm{~J}_{8.0}, 2 \mathrm{H}, \mathrm{H}-2 \text { ', 6’) }\right. \\
6.95-7.22(\mathrm{~m}, 4 \mathrm{H}, \mathrm{H}-2 ” \\
\left.3^{\prime}, 5^{\prime}, 6^{\prime \prime}\right)\end{array}$ & $\begin{array}{l}5.91 \\
\left(\mathrm{~d}, \mathrm{~J}_{4.1}, 1 \mathrm{H}\right)\end{array}$ & $\begin{array}{l}4.45 \\
\left(\mathrm{~d}, \mathrm{~J}_{4.1}, 1 \mathrm{H}\right)\end{array}$ & $\begin{array}{l}3.70 \\
(\mathrm{Ar}- \\
\left.\mathrm{OC}_{3}{ }_{3}\right)\end{array}$ & $\cdots$ \\
\hline $3 e$ & $\begin{array}{l}10.93 \\
(\mathrm{~s}, 1 \mathrm{H}, \mathrm{N} \underline{\mathrm{H}})\end{array}$ & $\begin{array}{l}10.42 \\
(\mathrm{~s}, 1 \mathrm{H}, \mathrm{N} \underline{\mathrm{H}})\end{array}$ & $\begin{array}{l}7.68\left(\mathrm{~d}, \mathrm{~J}_{8.2}, 2 \mathrm{H}, \mathrm{H}-2^{\prime}, 6^{\prime}\right) \\
7.39\left(\mathrm{~d}, \mathrm{~J}_{8.2}, 2 \mathrm{H}, \mathrm{H}-3{ }^{\prime}, 5^{\prime}\right) \\
7.09-7.14(\mathrm{~m}, 5 \mathrm{H}, \mathrm{H}- \\
\text { 2”,3”, 4”,5”,6”) }\end{array}$ & $\begin{array}{l}5.98 \\
(\mathrm{bs}, 1 \mathrm{H})\end{array}$ & $\begin{array}{l}4.41 \\
(\mathrm{bs}, 1 \mathrm{H})\end{array}$ & $\begin{array}{l}2.24 \\
(\mathrm{Ar}- \\
\left.\mathrm{CH}_{3}\right)\end{array}$ & $\ldots$ \\
\hline $3 f$ & $\begin{array}{l}12.66 \\
(\mathrm{~s}, 1 \mathrm{H}, \mathrm{N} \underline{\mathrm{H}})\end{array}$ & $\begin{array}{l}12.05 \\
(\mathrm{~s}, 1 \mathrm{H}, \mathrm{N} \underline{\mathrm{H}})\end{array}$ & $\begin{array}{l}7.70\left(\mathrm{~d}, \mathrm{~J}_{8.3}, 2 \mathrm{H}, \mathrm{H}-2^{\prime}, 6^{\prime}\right) \\
7.42\left(\mathrm{~d}, \mathrm{~J}_{8.3}, 2 \mathrm{H}, \mathrm{H}-3{ }^{\prime}, 5^{\prime}\right) \\
7.02-7.19(\mathrm{~m}, 5 \mathrm{H}, \mathrm{H}- \\
\text { 2”,3”, 4”,5”,6”) }\end{array}$ & $\begin{array}{l}5.95 \\
(\mathrm{bs}, 1 \mathrm{H})\end{array}$ & $\begin{array}{l}4.60 \\
(\mathrm{bs}, 1 \mathrm{H})\end{array}$ & $\begin{array}{l}2.26 \\
(\mathrm{Ar}- \\
\left.\mathrm{CH}_{3}\right)\end{array}$ & $\ldots$ \\
\hline $3 g$ & $\begin{array}{l}12.39 \\
(\mathrm{~s}, 1 \mathrm{H}, \mathrm{N} \underline{\mathrm{H}})\end{array}$ & $\begin{array}{l}12.15 \\
(\mathrm{~s}, 1 \mathrm{H}, \mathrm{N} \underline{\mathrm{H}})\end{array}$ & $\begin{array}{l}8.30\left(\mathrm{~d}, \mathrm{~J}_{7.9}, 2 \mathrm{H}, \mathrm{H}-2^{\prime}, 6^{\prime}\right) \\
7.85\left(\mathrm{~d}, \mathrm{~J}_{7.9}, 2 \mathrm{H}, \mathrm{H}-3{ }^{\prime}, 5^{\prime}\right) \\
6.98-7.29(\mathrm{~m}, 4 \mathrm{H}, \mathrm{H}- \\
\left.2 ”, 3 ”, 5 ”, 6^{\prime}\right)\end{array}$ & $\begin{array}{l}5.99 \\
\left(\mathrm{~d}, \mathrm{~J}_{3.9}, 1 \mathrm{H}\right)\end{array}$ & $\begin{array}{l}4.55 \\
\left(\mathrm{~d}, \mathrm{~J}_{3.9}, 1 \mathrm{H}\right)\end{array}$ & $\begin{array}{l}2.35 \\
(\mathrm{Ar}- \\
\left.\mathrm{CH}_{3}\right)\end{array}$ & $\cdots$ \\
\hline
\end{tabular}


1640-1491 $\mathrm{cm}^{-1}$ were assigned to $\mathrm{C}=\mathrm{C}$ of aromatic rings and $\mathrm{C}=\mathrm{N}$ of the conjugated form of barbituric acid part. Additional bands were observed at $1463-751 \mathrm{~cm}^{-1}$ due to these structural units (Bojarski et al., 1985) including bands for C-O-C at 1151-1078 $\mathrm{cm}^{-1}$.

The N-H protons at positions 1 and 3 in the compounds 3a-g were strongly deshielded ( $\delta$ 12.66-10.35) and appeared as singlet in their ${ }^{1} \mathrm{H}$ NMR spectra (Table 3 ). The $\mathrm{N}-\mathrm{H}$ protons at position 3 in these compounds were found comparatively more deshielded than protons at position 1 . In some compounds (3a, $\mathbf{3 c}, \mathbf{3} \mathbf{f}$ \& 3g) more deshielding of the $\mathrm{N}-\mathrm{H}$ protons were observed due to presence of thiocarbonyl group. This may be attributed to the greater polarizability of sulfur in comparison to oxygen. The proton at position 6 in 3a-g appeared as a doublet (or broad singlet) due to the vicinal coupling with the proton at position 5 . The chemical shifts were observed at $\delta 5.99-5.88$. The $5-\mathrm{H}$ in these compounds gave signals at $\delta 4.60-4.40$ as doublet due to the coupling received from the proton at position 6. The chemical shifts for the aromatic protons in $\mathbf{3 a - g}$ were found in good agreement with the literature values (Silverstein et al., 1991; Kemp 1991).

The structures of the compounds 3a-g were further confirmed by their ${ }^{13} \mathrm{C}$ NMR spectra (Table IV). The chemical shifts of carbonyl carbons at 4-C were found to be deshielded in the range of $\delta$ 165.40-160.96. The chemical shifts of 9-C were also deshielded ( $\delta$ 158.07-154.35). This value is comparable with the ${ }^{13} \mathrm{C}$ NMR chemical shifts of cyclohexyl methyl ketone (Marr et al., 1965).

\section{Table IV. ${ }^{13} \mathrm{C}$ NMR spectral data of the compounds 3a-g. [(d) in ppm]}

\begin{tabular}{|c|c|c|c|c|c|c|c|c|c|c|}
\hline Compound & $4-\mathrm{C}$ & $9-\mathrm{C}$ & $7-\mathrm{C}$ & $2-\mathrm{C}$ & $\begin{array}{l}\text { Aromatic } \\
\text { carbons }\end{array}$ & $6-\mathrm{C}$ & $10-\mathrm{C}$ & $5-\mathrm{C}$ & $\mathrm{X}$ & $\mathrm{Y}$ \\
\hline $3 \mathbf{a}$ & 160.96 & 158.07 & 153.59 & 173.70 & $144.94-113.80$ & 103.26 & 92.92 & 33.91 & $\begin{array}{l}55.07 \\
(\mathrm{Ar}- \\
\left.\mathrm{OCH}_{3}\right)\end{array}$ & $\begin{array}{l}20.79 \\
(\mathrm{Ar}- \\
\left.\underline{\mathrm{CH}_{3}}\right) \\
\end{array}$ \\
\hline $3 \mathbf{b}$ & 163.36 & 154.35 & 144.18 & 143.42 & $144.04-113.15$ & 104.26 & 92.82 & 34.01 & $\begin{array}{l}55.71 \\
(\mathrm{Ar}- \\
\left.\mathrm{OCH}_{3}\right)\end{array}$ & $\begin{array}{l}20.80 \\
(\mathrm{Ar}- \\
\left.\mathrm{CH}_{3}\right)\end{array}$ \\
\hline $3 c$ & 161.96 & 155.43 & 145.01 & 173.70 & $145.92-114.10$ & 103.72 & 88.45 & 32.85 & $\begin{array}{l}56.10 \\
(\mathrm{Ar}- \\
\left.\mathrm{OCH}_{3}\right)\end{array}$ & $\ldots$ \\
\hline 3d & 163.10 & 155.41 & 145.91 & 143.70 & $144.44-114.00$ & 103.90 & 89.35 & 32.82 & $\begin{array}{l}56.15 \\
(\mathrm{Ar}- \\
\left.\mathrm{OCH}_{3}\right)\end{array}$ & $\ldots$ \\
\hline $3 e$ & 163.02 & 155.08 & 149.63 & 144.59 & $139.79-125.97$ & 102.67 & 85.94 & 32.44 & $\begin{array}{l}21.13 \\
(\mathrm{Ar}- \\
\left.\mathrm{CH}_{3}\right)\end{array}$ & $\ldots$ \\
\hline $3 f$ & 163.20 & 155.80 & 148.61 & 174.59 & $139.80-125.51$ & 102.62 & 86.15 & 32.40 & $\begin{array}{l}20.97 \\
(\mathrm{Ar}- \\
\left.\underline{\mathrm{CH}}_{3}\right)\end{array}$ & $\ldots$ \\
\hline $3 g$ & 165.40 & 154.50 & 148.60 & 175.59 & $149.53-123.77$ & 105.65 & 89.65 & 34.44 & $\begin{array}{l}21.70 \\
(\mathrm{Ar}- \\
\left.\mathrm{CH}_{3}\right)\end{array}$ & $\ldots$ \\
\hline
\end{tabular}


The chemical shifts of carbonyl carbons at 2-C in the compounds $\mathbf{3 b}, \mathbf{3 d}$ and $\mathbf{3 e}$ were found to be at $\delta$ 144.59-143.42 and are relatively less deshielded due to the resonance of amide functional group. The chemical shifts of thioxo carbon at 2-C in the compounds $\mathbf{3 a}, \mathbf{3 c}, \mathbf{3 f}$ and $\mathbf{3 g}$ were found to be at $\delta 175.59-173.70$. This explains that the replacement of a carbonyl group by a thiocarbonyl group results in a downfield shift (Otto et al., 1976; Ahmed et al., 2005).

The chemical shift values for 7-C and 6-C in these compounds were observed at 153.59-144.18 and $\delta$ 105.65-102.62 respectively. The 10-C of the compounds showed chemical shift values at $\delta$ 92.92-85.94 which were comparable to the earlier report (Bojarski et al., 1985) of the ${ }^{13} \mathrm{C}$ NMR spectral data of the monosubstituted barbiturates at $10-\mathrm{C}$. The chemical shift values for 5-C in these compounds were observed at $\delta 34.44-32.40$.

The ${ }^{13} \mathrm{C}$ NMR chemical shifts for the carbons of aromatic rings were assigned on the basis of a correlation chart available in the literature (Levy et al., 1972).

The compounds 3a-g showed peaks for their respective molecular ions $\left(\mathrm{M}^{+}\right)$in their high resolution mass spectra at $\mathrm{m} / \mathrm{z} 378.14(47 \%), 362.20(10.7 \%), 398.06(15 \%)$, $382.19(13.0 \%), 332.25(40.5 \%), 348.12(10.3 \%)$ and $393.22(9.0 \%)$ respectively. The isotopic pattern for $\mathrm{Cl}$ atom $\left({ }^{35} \mathrm{Cl} /{ }^{37} \mathrm{Cl}, 3: 1\right)$ was observed in the molecular mass of the compounds $\mathbf{3} \mathbf{c}$ and $\mathbf{3 d}$.

\section{Acknowledgement}

Authors gratefully acknowledge the help of Prof. Teruo Shinmyozu, Department of Molecular Chemistry, Kyushu University, Fukuoka, Japan for recording proton and carbon-13 nmr spectra, mass spectra and determining elemental analyses of the compounds.

\section{References}

Ahmed MG, Ahmed SA, Ahmed SM, Hussam A and Hossain MM (2005), Synthesis of $7,11-\mathrm{d}$ i a r y $1-2,4-\mathrm{d}$ i a z a s p i r o $[5,5]$ undecane-3-oxo(orthioxo)-1,5,9-trines, Part-1., J. Chem. Res. 10: 622-625.
Akhter K, Ahmed SM, Halim ME, Kader MA, Jahan K, Romman UKR and Ahmed MG (2016), One step cyclocondensation of (thio)barbituric acid with chalcones in glacial acetic acid and phosphorous pentoxide, Part-I., Bangladesh J. Sci. Ind. Res. 51(2): 129-138.

Ahlwalia VK, Aggarwal R and Kumar R (1993), A Convenient One-Pot Synthesis of 5 - A ry l- 7 - met hy 1-1,2,3,4-tetrahydro-2, 4-dioxo-5H-pyrano(2,3-d) pyrimidines, Ind. $J$. Chem. 32B: 963-964.

Ahmed MG, Romman UKR, Ahmed SM, Akhter K, Halim ME and Salauddin M (2006), A study on the synthesis o f 5,7-diary 1-1,2,3,4-tetrahydro-2, 4-dioxo-5H-pyrano [2,3-d] pyrimidines, Bangladesh J. Sci. Ind. Res. 41(3-4): 119-128.

Ahmed MG, Romman UKR, Akhter K, Halim ME, Rahman MM and Ahmed SM (2011), A one-step synthesis of 5,7-Diaryl-1, 5-dihydro (or 1,2,3, 5-tetrahydro)-pyrano $[2,3-d]$ pyrimidin-2,4-diones (or 2-thioxo-4-ones), Ind. J. Chem. 50B: 946-948.

Bararjanian M, Balalaie S, Movassagh B and Amani AM (2009), One-Pot Synthesis of Pyrano [2,3-d]pyrimidinone Derivatives Catalyzed by L-Proline in Aqueous Media, J. Iran. Chem. Soc. 6(2): 436-442.

Bojarski JT, Mokrosz JL, Barton HJ and Paluchowaka MH (1985), Advances in Heterocyclic Chemistry (Review Article), 38: 229-297.

Broom AD, Shim JL and Anderson GL (1976), Pyrido [2,3-d]pyrimidines. IV. Synthetic studies leading to various oxopyrido [2,3-d] pyrimidines, J Org Chem. 41: 1095-1099.

Coates WJ (1990), Pyrimidopyrimidine Derivatives, Eur Pat 351058, Chem Abstr 113: 40711.

Furuya S and Ohtaki T (1994), Pyridopyrimidine derivatives, their production and use, Eur Pat Appl EP 608565, Chem Abstr 121: 205395w. 
Furniss BS, Hannaford AJ, Rogers V, Smith PWG and Tatchell AR (1978), Vogel's Text Book of Practical Organic Chemistry, $4^{\text {th }}$ Ed., Longman Group Ltd., London, p 796.

Heber D, Heers C and Ravens U (1993), Positive inotropic activity of 5-amino-6-cyano-1, 3-dimethyl-1, 2, 3, 4-tetrahydropyrido [2, 3-d] pyrimidine-2, 4-dione in cardiac muscle from guinea-pig and man. Part 6: compounds with positive inotropic activity, Die Pharmazie 48: 537-541.

Junek H and Aigner H (1973), Synthesen mit Nitrilen, XXXV. Reaktionen von Tetracyanäthylen mit Heterocyclen, Chem Ber. 106: 914-921.

Kitamura N and Onishi A (1984), Eur. Pat. 163599, Chem Abstr 104: 186439.9

Kharchenko VG, Markova LI and Korshunova KM (1976), On Reactions of oxo-1, 5-diketones with sulfurous reagents, Zh. Org. Khim. 12(3): 663; Chem Abstr 85: 32775c.

Kemp W (1991), Organic Spectroscopy, $3^{\text {rd }}$ Ed., Macmillan, London.

Levy GC and Nelson GL (1972), Carbon-13 Nuclear Magnetic Resonance for Organic Chemists, John Wiley \& Sons, N. Y.

Levitt G (1982), Herbicidal sulfonamides, US Patent 4339267; Chem Abstr 98: 215602g.

Marr DH and Stothers JB (1965), ${ }^{13}$ C NMR Studies: Part VI. Carbon-13 Spectra of $\alpha, \beta$ - Unsaturated Carbonyl Compounds, Canad. J. Chem. 43: 596-607.

Noboru S, Yoshikazu K and Psurematsu T (1973), Synthesis of 5-Pyrazolylbarbituric Acid Derivatives, Chem. Pharm. Bull. 21(12): 2639-2642.

O’Callaghan CN and Conalty ML (1983), Anticancer Agents: XVII. Synthesis and antitumour activity of 2-uyl-4-oxo-2,3-dihydrobenzopyrano[2,3-d] pyrimidines, and 4-substituted 2-aryl-5h-benzopyrano-[2,3-d] pyrimidines, Proc. R. Ir. Acad. 83B: 241.

Otto HH and Triepel J (1976), Synthesis and structure of 7,11-diphenyl-2,4-diazaspiro[5.5] undecan-1,3,5,9-tetraones, I. Liebigs Ann. Chem. 1982-1991.

Rao AS and Mitra RB (1974), Synthesis of heterocycles. II. Pyrano [2,3-dlpyrimidines, Ind. J. Chem. 12: 1028; Chem Abstr 82: 112023.

Rahman MM, Ahmed SM, Siddiki SMAH, Halim ME, Akhter K, Romman UKR and Ahmed MG (2013), A one pot synthesis of 5, 7-diaryl-1,5-dihydro (or $1, \quad 2, \quad 3, \quad 5$-tetrahydro)- $\operatorname{pyrano}[2,3-d]$ pyrimidin-2, 4-diones (or 2-thioxo-4-ones), Dhaka Univ. J. Sci. 61(2): 167-171.

Senda S, Fujimura H and Izumi H (1968), Barbituric acid analgesics, Japan Patent 193, 6824; Chem Abstr 70: $78001 \mathrm{r}$.

Silverstein RM, Bassler GC and Morill TC (1991), Spectroscopic Identification of Organic Compounds, $5^{\text {th }}$ Ed., John Wiley \& Sons, N.Y.

Tietze LF and Kettschau G (1997), Topics in Current Chemistry, Springer, Berlin 189: 1-120.

Ullah MS, Ahmed MG, Romman UKR, Akhter K, Ahmed SM and Halim ME (2012), Synthesis of 5, 7-diaryl-1, 5-dihydro (or 1, 2, 3, 5-tetrahydro)pyrano $[2,3-d]$ pyrimidin-2, 4-diones (or 2-thioxo-4-ones), J. Bangladesh Chem. Soc. 25(2): 124-131.

Wrigglesworth $\mathrm{R}$, Inglish WD, Livingstone DB, Suekling CJ and Wood HCS (1984), Specific enzyme inhibitors in vitamin biosynthesis. Part 6. Identification of an affinity chromatography ligand for the purification of riboflavin synthase, J. Chem. Soc. (Perkin Trans I) 5: 959-963. 
Ziarani GM, Faramarzi S, Asadi S, Badiei A, Bazl R and Amanlou M (2013), Three-component synthesis of pyrano $[2,3-d]$-pyrimidine dione derivatives facilitated by sulfonic acid nanoporous silica
$\left(\mathrm{SBA}-\mathrm{Pr}-\mathrm{SO}_{3} \mathrm{H}\right)$ and their docking and urease inhibitory activity, DARU Journal of Pharmaceutical Sciences 21: 3.

Received: 28 May 2016; Revised: 5 June 2016 ; Accepted: 28 December 2016. 